\title{
Tangle Decompositions of Doubled Knots
}

\author{
Hiroshi MATSUDA \\ University of Tokyo \\ (Communicated by S. Suzuki)
}

\begin{abstract}
We show that a decomposing 2-sphere which gives an essential tangle decomposition of a doubled knot gives also an essential tangle decomposition of its companion knot.
\end{abstract}

\section{Introduction.}

The notion of doubled knots was introduced by J. H. C. Whitehead in [W]. Recall the definition of the doubled knot $K$ of a knot $K_{1}$. Let $V_{0} \subset S^{3}$ be an unknotted solid torus and $J$ a simple closed curve in $V_{0}$, as shown in Figure 1. Let $V_{1}$ be a tubular neighborhood of $K_{1}$ and $h: V_{0} \rightarrow V_{1}$ a homeomorphism with $h\left(m_{0}\right)=m_{1}$ and $h\left(l_{0}\right)=l_{1}+q \cdot m_{1}$, where $\left(m_{i}, l_{i}\right)\left(\subset \partial V_{i}\right)$ is a meridian-longitude pair for $V_{i}(i=0,1)$ and $q$ is an integer. Then we say $K=h(J)$ the doubled knot of $K_{1}$. The knot $K_{1}$ is said to be the companion knot of $K$ constructed in this manner up to knot type.

Let $B$ be a 3-ball and $t=t_{1} \cup \cdots \cup t_{n}$ a union of mutually disjoint $n$ arcs properly embedded in $B$. Then the pair $(B, t)$ is said to be an $n$-string tangle. We say that an $n$-string tangle $(B, t)$ is trivial if $(B, t)$ is homeomorphic to $\left(D \times I,\left\{x_{1}, \cdots, x_{n}\right\} \times I\right)$ as pairs, where $D$ is a 2-disc and $x_{i}$ is a point in $\operatorname{Int} D(i=1, \cdots, n)$. Let $N(t)$ denote a regular neighborhood of $t$ in $B$. The tangle $(B, t)$ is said to be essential if $c l(\partial B-N(t))$ is incompressible in $\operatorname{cl}(B-N(t))$ and $(B, t)$ is not a trivial 1-string tangle. If $\left(S^{3}, K\right)=$ $\left(B_{1}, t_{1}\right) \cup\left(B_{2}, t_{2}\right)$ and $\left(B_{i}, t_{i}\right)$ is an essential $n$-string tangle for $i=1$ and 2 , then it is said that a knot $K$ in $S^{3}$ admits an essential $n$-string tangle decomposition. If a knot $K$ admits no essential $k$-string tangle decomposition, then $K$ is said to be $k$-string prime.

Then our results are;

THEOREM. If a doubled knot $K$ admits an essential $m$-string tangle decomposition, then $m$ is even, and this decomposing 2-sphere gives an essential $m / 2$-string tangle decomposition of the companion knot.

COROllary. A doubled knot whose companion is $n$-string prime for every $n \geq 1$ is also $m$-string prime for every $m \geq 1$.

Received February 3, 1997

Revised July 15, 1997 


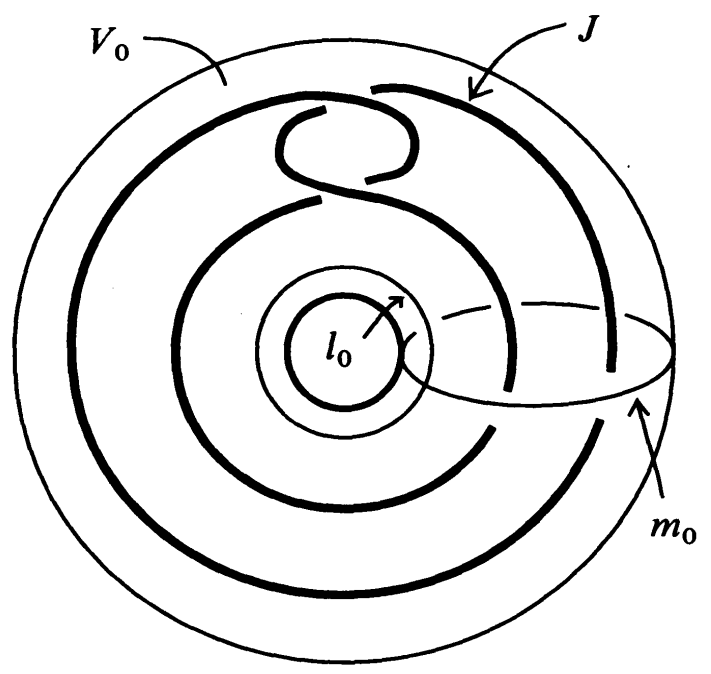

FIGURE 1

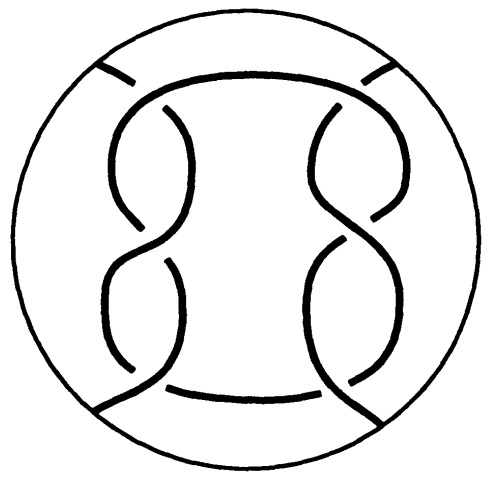

$(B, t)$

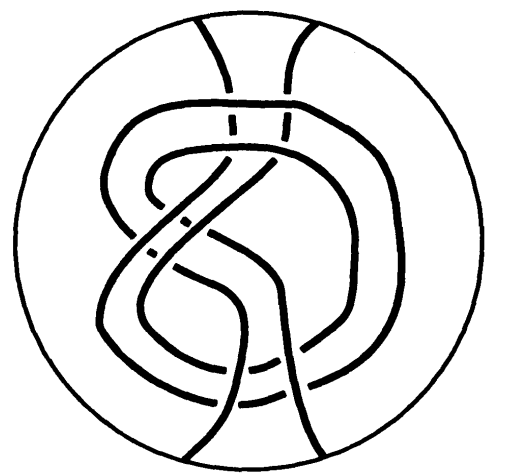

$(C, s)$

(a)

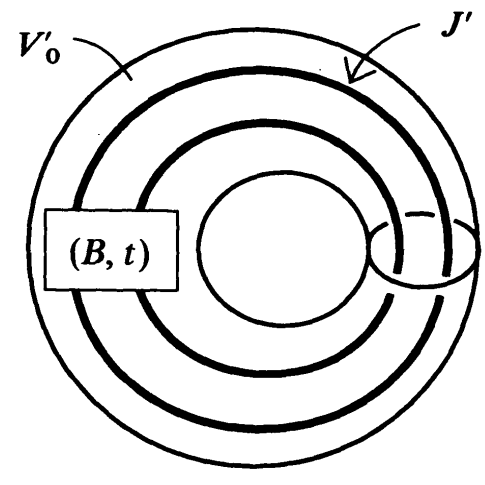

(b)

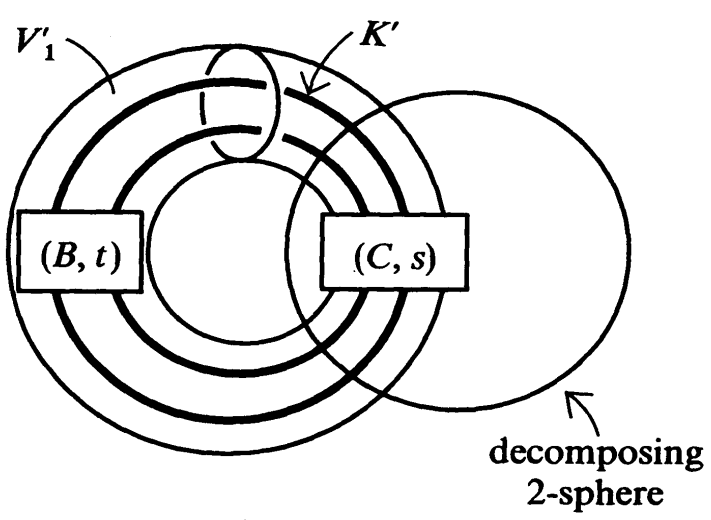

(c)

Figure 2 
Proof of Corollary. By the above Theorem, a doubled knot is $m$-string prime for every odd $m$. The companion knot is $n$-string prime for every $n \geq 1$, so its doubled knots are $m$-string prime for every even $m$.

We note here that the theorem is not valid for arbitrary satellite knots and links which are 1-string prime.

Let $(B, t),(C, s)$ be essential 2-string tangles, for example, see Figure 2 (a). The diagram $(C, s)$ represents a knotted spanning arc of the ball and another arc running parallel to it. The figure shows the arcs knotted in trefoil knot, but any knot will suffice. For the essentiality of these tangles, see [B] or [L].

Let $J^{\prime}$ be a knot or link in an unknotted solid torus $V_{0}^{\prime}$ obtained from a tangle $(B, t)$, as shown in Figure 2 (b). Let $V_{1}^{\prime}$ be a tubular neighborhood of a knot $K_{1}^{\prime}$. In the same way as we construct a doubled knot, we construct a satellite link $K^{\prime}=h\left(J^{\prime}\right)$. The companion of $K^{\prime}$ is $K_{1}^{\prime}$. From the way of construction, we assume that $\left(S^{3}, K^{\prime}\right)=(B, t) \cup(C, s)$, as shown in Figure 2 (c).

Then the decomposing 2-sphere which realizes an essential 2-string tangle decomposition of $K^{\prime}$ gives an inessential 1-string tangle decomposition of the companion. See Figure 2 (c). For the 1-string primeness of the resulting knot and link, see [B] or [L].

\section{Reducing the number of intersections.}

Let $K$ be a doubled knot in $S^{3}$ and $D$ a 2-disc. Then there exists an immersion $f: D \rightarrow S^{3}$ such that $\left.f\right|_{\partial D}: \partial D \rightarrow K$ is a homeomorphism and $f^{-1}(\tilde{\Sigma})$ consists of two arcs $\sigma_{1}, \sigma_{2}$ joining a point in $\partial D$ and a point in $\operatorname{Int} D \cap f^{-1}(K)$, where $\tilde{\Sigma}=$ $\left\{x \in f(D)|| f^{-1}(x) \mid \geq 2\right\}$.

Put $\tilde{D}=f(D), \quad \Sigma=f^{-1}(\tilde{\Sigma})$ and then $\Sigma=\sigma_{1} \cup \sigma_{2}$. Put $\partial \sigma_{i} \cap \operatorname{Int} D=\left\{x_{i}\right\}, \partial \sigma_{i} \cap$ $\partial D=\left\{y_{i}\right\}$

Suppose that $K$ admits an essential $m$-string tangle decomposition, then there exists a 2-sphere $S$ in $S^{3}$ which realizes this decomposition of $\left(S^{3}, K\right)$. We may assume that $S$ intersects $\tilde{D}$ transversely. Put $\widetilde{C}=S \cap \tilde{D}$ and $C=f^{-1}(\widetilde{C})$, then $C$ consists of $m$ arcs properly embedded in $D$ and simple loops in Int $D$.

We now introduce a measure of the complexity of the pair $(\tilde{D}, S)$. Let $\left|f\left(\sigma_{1}\right) \cap S\right|$ be the number of points in $f\left(\sigma_{1}\right)\left(=f\left(\sigma_{2}\right)\right) \cap S,|C(l)|$ the number of loops in $C$. The complexity $c(\tilde{D}, S)$ is the pair $\left(\left|f\left(\sigma_{1}\right) \cap S\right|,|C(l)|\right)$. We assign the standard lexicographic ordering to this complexity function.

We may assume that $c(\tilde{D}, S)$ is minimum among all 2-spheres ambient isotopic in the pair $\left(S^{3}, K\right)$ to $S$.

We will prove the theorem by showing that $C$ consists of parallel arcs on $D$ each of which separates $x_{1}$ from $x_{2}$ and is disjoint from $\sigma_{1}$ and $\sigma_{2}$.

Lemma 1. A loop component $\alpha$ of $C$ bounds a disc $D^{\prime}$ in $D$ with $\partial D^{\prime}=\alpha$, $D^{\prime} \cap\left\{x_{1}, x_{2}\right\} \neq \varnothing$. 
Proof. Without loss of generality we assume that $\alpha$ is an innermost loop component of $C$ in $D$. We assume for a contradiction that $D^{\prime} \cap x_{1}=D^{\prime} \cap x_{2}=\varnothing$. If $\alpha \cap \operatorname{Int} \sigma_{i} \neq \varnothing$, then there are subarcs $\gamma$ of $\sigma_{i}$ and $\gamma^{\prime}$ of $\partial D^{\prime}$ such that $\partial \gamma^{\prime}=\partial \gamma$ and $\gamma$ is outermost in $D^{\prime}$, that is, the arcs $\gamma$ and $\gamma^{\prime}$ cobound a disc $\delta$ in $D^{\prime}$ such that (Int $\left.\delta\right) \cap \sigma_{i}=\varnothing$. We may then isotope $S$ along $\delta$ to reduce the number of intersections of $\sigma_{i}$ with $S$.

Hence we may assume $\alpha \cap \sigma_{i}=\varnothing$. Let $E_{1}$ and $E_{2}$ be two discs in $S$ separated by $\alpha$, and put $S_{i}=E_{i} \cup D^{\prime}$. Let $B_{i}$ be the 3-ball in $S^{3}$ bounded by $S_{i}$ with $B_{1} \cap B_{2}=D^{\prime}$, and $t=\left(B_{1} \cup B_{2}\right) \cap K$. If $E_{i} \cap K \neq \varnothing$ for $i=1$ and 2, then the disc $D^{\prime}$ implies that $\operatorname{cl}(S-N(t))$ is compressible in $\operatorname{cl}\left(B_{1} \cup B_{2}-N(t)\right)$, and violates the essentiality of the tangle. If either $E_{1} \cap K=\varnothing$ or $E_{2} \cap K=\varnothing$, then either $B_{1} \cap K$ or $B_{2} \cap K$, say $B_{2} \cap K$, is an empty set. Then by isotoping $S$ along the ball $B_{2}$ we obtain the 2 -sphere $S_{1}$, and we further isotope $S_{1}$ slightly off from $D^{\prime}$ to reduce the intersection loop $\alpha$. This is a contradiction.

Let $\gamma$ be an outermost arc component of $C$ in $D$ and $\gamma^{\prime}$ a subarc in $\partial D$ with $\partial \gamma^{\prime}=\partial \gamma$ such that the loop $\gamma \cup \gamma^{\prime}$ bounds a disc $\delta$ in $D$ and (Int $\left.\delta\right) \cap S$ contains no arc component of $C$.

LEMMA 2. The disc $\delta$ contains $x_{1}$ or $x_{2}$.

Proof. Suppose $\delta$ contains neither $x_{1}$ nor $x_{2}$.

We may assume that $B_{k}(k=1$ or 2$)$ contains $\delta$. Let $N\left(\delta ; B_{k}\right)$ denote a regular neighborhood of $\delta$ in $B_{k}$, and $t=K \cap B_{k}$. If $\delta$ does not contain a subarc of $\sigma_{1}, \sigma_{2}$, then the disc $c l\left(\partial N\left(\delta ; B_{k}\right)-S\right)$ implies that $c l\left(\partial B_{k}-N(t)\right)$ is compressible in $c l\left(B_{k}-N(t)\right)$, and violates the essentiality of the tangle.

There exist two types of subarcs of $\sigma_{1}, \sigma_{2}$ in $\delta$. One type is a subarc $\sigma^{1}$ whose endpoints are in $\gamma$. Without loss of generality we may suppose that $\sigma^{1}$ is outermost in $\delta$, that is, there is a subarc $\gamma^{1}$ of $\gamma$ with $\partial \sigma^{1}=\partial \gamma^{1}$, and the arcs $\sigma^{1}$ and $\gamma^{1}$ cobound a disc $\delta^{1}$ in $\delta$ such that $\delta^{1}$ contains no arc component. We may then isotope $S$ along $\delta^{1}$ to reduce the number of intersections of $\sigma_{i}$ with $S$. This contradicts the minimality of $c(\tilde{D}, S)$. Hence, in fact, there are no arc of this type.

The other type is a subarc $\sigma^{2}$, one of whose endpoints is in $\gamma$, the other in $\gamma^{\prime}$. Without loss of generality we may assume that $\sigma^{2}$ is outermost in $\delta$, because there are no subarcs of $\sigma_{i}$ whose endopoints are in $\gamma$. There is a subarc $\gamma^{2}$ of $\partial \delta$ with $\partial \sigma^{2}=\partial \gamma^{2}$, and the arcs $\sigma^{2}$ and $\gamma^{2}$ cobound a disc $\delta^{2}$ in $D_{i}$ such that $\delta^{2}$ contains no arc component. We may then isotope $S$ along $\delta^{2}$ to reduce the number of intersections of $\sigma_{i}$ with $S$. This contradicts the minimality of $c(\tilde{D}, S)$.

By Lemma 2, the arc component of $C$ are mutually parallel. That is, each $\gamma$ separates $x_{1}$ and $x_{2}$. Call these arcs $\gamma_{1}, \gamma_{2}, \cdots, \gamma_{m}$. These arcs separate $D$ into $m+1$ discs, say $D_{0}, D_{1}, \cdots, D_{m}$ as shown in Figure 3. The discs $D_{0}, D_{m}$ are the outermost discs.

LEMMA 3. $\sigma_{i} \subset D_{0} \cup D_{m}(i=1$ and 2$)$.

Proof. Suppose $\sigma_{i}$ meets $\gamma_{1} \cup \cdots \cup \gamma_{m}$. We are in the situation in Figure 3. 


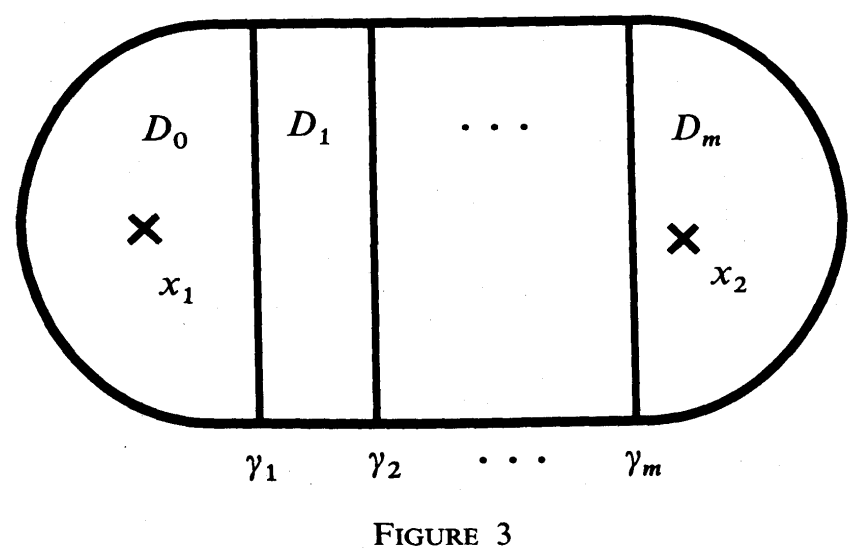

If the endpoint $y_{i}$ of $\sigma_{i}$ is in $\partial D_{0}$, then a subarc $\sigma^{1}$ of $\sigma_{i}$ connects $y_{i}$ and a point in $\gamma_{1}$ in $D_{0}$ because $\sigma_{i}$ is not contained in $D_{0}$. The arc $\sigma^{1}$ and a subarc of $\partial D_{0}$ cobound a disc $\delta^{1}$ in $D_{0}$, which contain neither $x_{1}$ nor $x_{2}$. Then we can show that we will decrease the complexity $c(\tilde{D}, S)$ in exactly the same way as we did in the proof of Lemma 2.

If $y_{i}$ is in $\partial D_{m}$, then we can show that we will decrease the complexity $c(\tilde{D}, S)$ in exactly the same way as we did in the proof of Lemma 2.

If $y_{i}$ is in $\partial D_{j}(j \neq 0, m)$, then a subarc $\sigma^{2}$ of $\sigma_{i}$ connects $y_{i}$ and a point in $\gamma_{j}$ or $\gamma_{j+1}$ in $D_{j}$. The arc $\sigma^{2}$ and a subarc of $\partial D_{j}$ which contains one subarc of $K$ cobound a disc $\delta^{2}$ in $D_{j}$. Then we can show that we can again decrease the complexity $c(\widetilde{D}, S)$ in exactly the same way as we did in the proof of Lemma 2.

Thus $\sigma_{i}$ does not meet $\gamma_{1} \cup \cdots \cup \gamma_{m}$, so we have $\sigma_{1} \subset D_{0}$ and $\sigma_{2} \subset D_{m}$.

Note that $\gamma_{1} \cup \cdots \cup \gamma_{m} \neq \varnothing$ because $S \cap K \neq \varnothing$. The remaining loops of $C$ are contained in $D_{0} \cup D_{m}$, and each loop meets $\sigma_{1}$ or $\sigma_{2}$. Put $p=\left|f\left(\sigma_{1}\right) \cap S\right|$ and $q=\left|f\left(\sigma_{2}\right) \cap S\right|$.

LEMMA 4. Each loop of $C$ meets $\sigma_{1}$ or $\sigma_{2}$ exactly once.

Proof. Suppose a loop $\alpha_{i}$ of $C$ which is contained in $D_{0}$ meets $\sigma_{1}$ more than once. Let $D_{i}^{\prime}$ be a disc in $D$ with $\partial D_{i}^{\prime}=\alpha_{i}$. The disc $D_{i}^{\prime}$ contains a subarc $\sigma^{i}$ of $\sigma_{1}$. The $\operatorname{arc} \sigma^{i}$ and a subarc of $\alpha_{i}$ cobound a disc $\delta^{i}$ in $D_{i}^{\prime}$ which does not contain $x_{1}$. Without loss of generality we may assume $\sigma^{i}$ is outermost in $D_{i}^{\prime}$, that is, $\operatorname{Int} \delta^{i}$ does not contain a subarc of $\sigma_{1}$. If the disc $\delta^{i}$ contains a subarc $c_{j}$ of $\alpha_{j}$, then both endpoints of $c_{j}$ are in $\sigma^{i}$. Note that $\alpha_{j}$ meets $\sigma_{1}$ more than once. Hence without loss of generality we may assume $\delta^{i} \cap \alpha_{k}=\varnothing$ for $k \neq i$, taking $\alpha_{i}$ to be an innermost one meeting $\sigma_{1}$ more than once.

Then we isotope $S$ along $\delta^{i}$ to reduce the number of intersections of $\sigma_{1}$ with $S$. This contradicts the minimality of $c(\tilde{D}, S)$. So we assume that each loop of $C$ in $D_{0}$ meets $\sigma_{1}$ exactly once.

In the same way as above, we may assume that each loop of $C$ contained in $D_{m}$ meets $\sigma_{2}$ exactly once. 


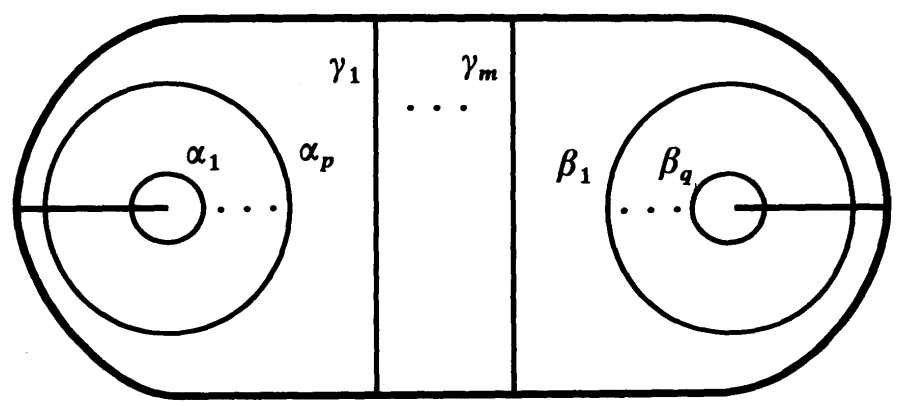

FIGURE 4

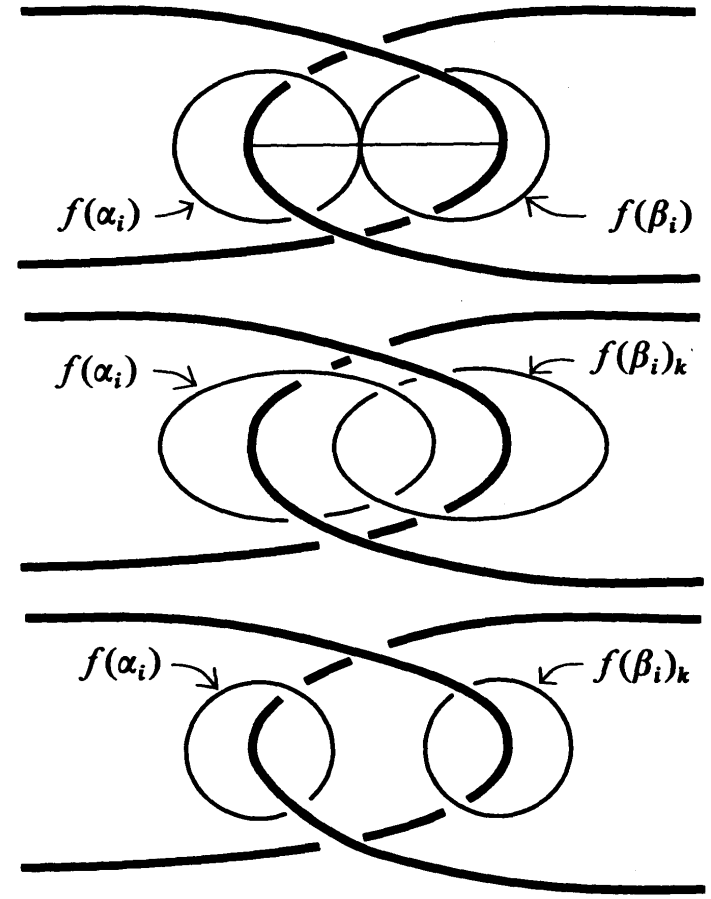

$(k=a$ or $b)$

FIGURE 5

We are now in the situation in Figure 4.

We call the loops in $D_{0} \alpha_{1}, \cdots, \alpha_{p}$ and those in $D_{m} \beta_{1}, \cdots, \beta_{q}$. Note that $p=q$ because $f\left(\sigma_{1}\right)=f\left(\sigma_{2}\right)$.

LEMMA 5. $p=q=0$.

Proof. Suppose $p \neq 0$. More $f\left(\beta_{i}\right)$ slightly off above (respectively, off below) the 2-sphere $S$, and call this $f\left(\beta_{i}\right)_{a}$ (resp. $\left.f\left(\beta_{i}\right)_{b}\right)$. Then one of $f\left(\alpha_{i}\right) \cup f\left(\beta_{i}\right)_{a}$ and $f\left(\alpha_{i}\right) \cup f\left(\beta_{i}\right)_{b}$ forms a Hopf link. See Figure 5.

On the other hand, since both $f\left(\alpha_{i}\right)$ and $f\left(\beta_{i}\right)$ are embedded in a common 2-sphere, both $f\left(\alpha_{i}\right) \cup f\left(\beta_{i}\right)_{a}$ and $f\left(\alpha_{i}\right) \cup f\left(\beta_{i}\right)_{b}$ are split links.

Thus we have a contradiction. 
By Lemma 5, we can see that if a doubled knot has an essential tangle decomposition, then this 2-sphere gives a (maybe inessential) tangle decomposition of the companion. In the following, we have to show that $m$ is even, and that this decomposition of the companion is essential.

Proof of Theorem. Suppose $m$ is odd.

Let $\kappa$ be a subarc of $\partial D$ with $\partial \kappa=\left\{y_{1}, y_{2}\right\}$. Then we can regard $f\left(\sigma_{1} \cup \kappa\right)$ as the companion of $K$, and it is a simple loop in $S^{3}$ which intersects $S$ transversely in odd points. This is a contradiction, so $m$ is even.

Suppose that the punctured sphere $c l(S-N(f(D)))$ has a compressing disc $Q$. Then this disc $Q$ will be a compressing disc for $\operatorname{cl}(S-N(K))$, and violates the essentiality of the original tangle.

Suppose that one of the tangles of the decomposition of the companion is a trivial 1 -string tangle. Then its original tangle is a trivial 2-string tangle, which contradicts the essentiality of the original tangle. This completes the proof.

ACKNOWLedGements. The author would like to thank Dr. Chuichiro Hayashi, Mr. Koya Shimokawa and Mr. Makoto Ozawa for their helpful comments and encouragements.

\section{References}

[B] S. A. BleiER, Knots prime on many strings, Trans. Amer. Math. Soc. 282 (1984), 385-401.

[L] W. B. R. Lickorish, Prime knots and tangles, Trans. Amer. Math. Soc. 267 (1981), 321-332.

[W] J. H. C. WhiteheAD, On doubled knots, J. London Math. Soc. 12 (1937), 63-71.

Present Address:

Graduate School of Mathematical Sciences, University of Tokyo,

Komaba, Meguro-Ku, TOKyo, 153-0041. 